\title{
An Oxygen Gettering Scheme for Improving Device Mobility and Subthreshold Swing of InGaZnO-Based Thin-Film Transistor
}

\author{
Hsiao-Hsuan Hsu, Chun-Yen Chang, Fellow, IEEE, Chun-Hu Cheng, Member, IEEE, Shan-Haw Chiou, \\ Chiung-Hui Huang, and Yu-Chien Chiu
}

\begin{abstract}
This study involved developing a low-power and highmobility metal-oxide thin-film transistor that incorporated a bilayer IGZO/IGZO:Ti semiconductor material. Compared with control metal-oxide TFTs, the bilayer IGZO TFT through thickness modulation of IGZO:Ti can reach the smallest subthreshold swing $(85 \mathrm{mV} /$ decade) and the highest field effect mobility $\left(49 \mathrm{~cm}^{2} / \mathrm{Vs}\right)$ at a drive voltage of $<3 \mathrm{~V}$. This performance level improvement can be attributed to the gettering effect caused by the IGZO:Ti capping layer and its dual advantages, which enhance device mobility and improve gate swing.
\end{abstract}

Index Terms-Gettering, InGaZnO (IGZO), thin-film transistor (TFT), titanium oxide $\left(\mathrm{TiO}_{x}\right)$.

\section{INTRODUCTION}

C OMPARED with poly-Si thin-film transistors (TFTs) that require a large thermal budget, amorphous $\mathrm{InGaZnO}$ $(\alpha$-IGZO) TFTs have drawn attention because they yield a larger driving current at a relatively low process temperature. An IGZO-driven active matrix organic light-emitting diode (AMOLED) has recently been applied to high-resolution displays [1]-[3]. Gate dielectrics that use materials with high dielectric constants have been proven to lower drive power levels when fabricating low-temperature TFTs [4]-[16]; however, IGZO TFTs continue to demonstrate high subthreshold swings and restricted device mobility, preventing them from being applied in fast-switching and high-resolution displays.

Therefore, a high-performance metal-oxide TFT was fabricated using a bilayer IGZO/IGZO:Ti channel structure. The IGZO:Ti capping layer that incorporates Ti can be used as a gettering layer to improve device mobility and transistor transfer characteristics. The transistor characteristics through thickness

Manuscript received March 1, 2014; revised June 4, 2014; accepted June 19, 2014. Date of publication June 23, 2014; date of current version September 4, 2014. This work was supported by the National Science Council of Taiwan under Contract NSC 102-2221-E-003-019. The review of this paper was arranged by Associate Editor J. Li.

H.-H. Hsu, C.-Y. Chang, and Y.-C. Chiu are with the Department of Electronics Engineering, National Chiao Tung University, Hsinchu 30010, Taiwan (e-mail: bluejerry723@hotmail.com; hhhsu@itri.org.tw; minos000000@ hotmail.com).

C.-H. Cheng is with the Department of Mechatronic Technology, National Taiwan Normal University, Taipei 10610, Taiwan (e-mail: chcheng@ ntnu.edu.tw)

S.-H. Chiou and C.-H. Huang are with the Material and Chemical Research Laboratory, Industrial Technology Research Institute, Hsinchu 31040, Taiwan (e-mail: raymondchengu@gmail.com; feldcheng@ hotmail.com).

Color versions of one or more of the figures in this paper are available online at http://ieeexplore.ieee.org.

Digital Object Identifier 10.1109/TNANO.2014.2332395

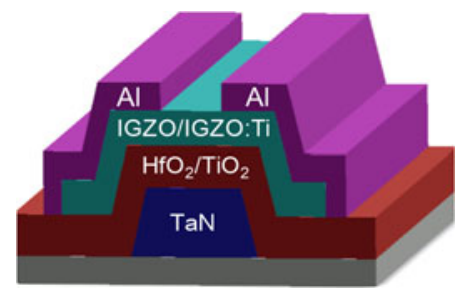

Fig. 1. Schematic structure of bilayer IGZO/IGZO:Ti TFT.

modulation of IGZO:Ti can reach the smallest subthreshold swing (SS; $85 \mathrm{mV} / \mathrm{decade})$ and the highest field effect mobility $\left(\mu_{\mathrm{FE}} ; 49 \mathrm{~cm}^{2} / \mathrm{Vs}\right)$ under a low drive voltage $\left(V_{G}-V_{T}\right)$ of $<3 \mathrm{~V}$.

\section{EXPERIMENTS}

A bottom-gate TFT was fabricated on 200-nm-thick insulating $\mathrm{SiO}_{2}$ substrate. A 35-nm-thick bottom TaN gate electrode was deposited using a sputtering system. Subsequently, 28-nm $\mathrm{HfO}_{2}$ and 26-nm $\mathrm{TiO}_{2}$ were deposited using electron beam evaporation and annealed at $400{ }^{\circ} \mathrm{C}$ in nitrogen to perform dielectric activation. Subsequently, 10-15-nm IGZO and 3-7-nm IGZO:Ti were deposited in a gas mixture of $30 \% \mathrm{O}_{2}$ in an $\mathrm{Ar}$ atmosphere to form bilayer IGZO/IGZO:Ti structures. Control channel layers of single-layer $\mathrm{TiO}_{x}$ and IGZO were also fabricated to facilitate comparison; these channel layers were annealed at $300{ }^{\circ} \mathrm{C}$ in nitrogen. The cation ratio of IGZO:Ti film $(\mathrm{In} / \mathrm{Ga} / \mathrm{Zn} / \mathrm{Ti}=24 / 18 / 12 / 46)$ was examined by $\mathrm{X}$-ray photoelectron spectroscopy. Finally, a 250-nm-thick Al film was evaporated to form source and drain contacts. The patterned channel size was $545 \mu \mathrm{m} \times 45 \mu \mathrm{m}$. The film stacks were analyzed using transmission electron microscopy (TEM). The electrical characteristics of the TFT devices were characterized using capacitance-voltage $(C-V)$ and current-voltage $(I-V)$ measurements, which were assessed using an HP4284A precision $L C R$ meter and HP 4156 semiconductor parameter analyzer, respectively. The self-consistent $a b$ initio calculations were also performed to investigate oxygen-vacancy model in bilayer IGZO/IGZO:Ti structure.

\section{RESUlTS AND DISCUSSION}

To investigate different channel materials, the TFT devices using IGZO, $\mathrm{TiO}_{2}$, and bilayer IGZO/IGZO:Ti TFT semiconductors were fabricated simultaneously. Fig. 1 shows the schematic 


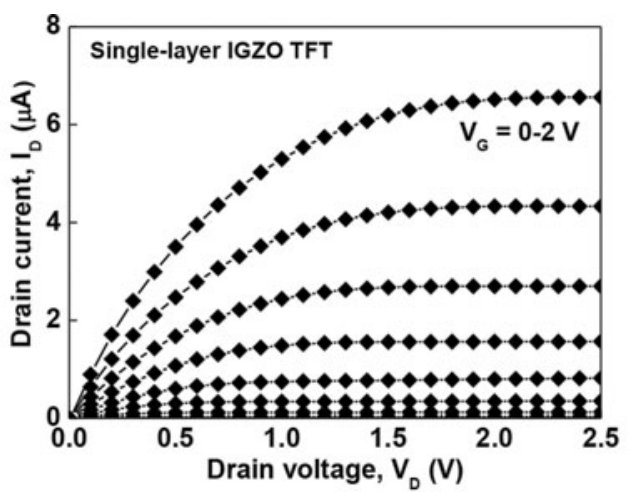

(a)

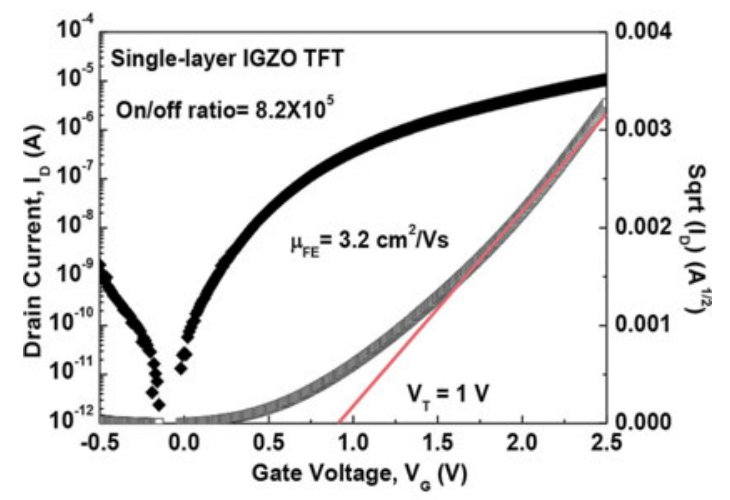

(b)

Fig. 2. (a) $I_{d}-V_{d}$ and (b) $I_{d}-V_{g}$ characteristics of IGZO TFT devices.

plot of bilayer IGZO/IGZO:Ti TFTs with $\mathrm{HfO}_{2} / \mathrm{TiO}_{2}$ gate dielectrics. The measured capacitance density of the stacked $\mathrm{HfO}_{2} / \mathrm{TiO}_{2}$ metal-insulator-metal capacitor is approximately $0.4 \mu \mathrm{F} / \mathrm{cm}^{2}$ at $100 \mathrm{kHz}$, yielding a high dielectric constant $(\kappa$ value) of 24. Compared to conventional silicon dioxide with a very small $\kappa$ value of 3.9, the high $\kappa$ value six times larger than $\mathrm{SiO}_{2}$ can reach lower gate leakage under an equivalent electrical field, which is helpful to reduce ac switching power. The stacked gate dielectric with large capacitance density and small capacitance equivalent thickness is also beneficial to lower drive voltage and gate swing.

Fig. 2(a) and (b) shows the output $\left(I_{d}-V_{d}\right)$ and transfer $\left(I_{d}-V_{g}\right)$ characteristics of a single-layer IGZO TFT device, respectively. The device performance shows an ON/OFF ratio of $8 \times 10^{5}$, a threshold voltage $\left(V_{T}\right)$ of $1 \mathrm{~V}, S S$ of $131 \mathrm{mV} /$ decade, and low $\mu_{\mathrm{FE}}$ of $3.2 \mathrm{~cm}^{2} / \mathrm{Vs}$. The high $\kappa$ gate dielectric reduces the operation voltage and $V_{T}$ [14]-[17]; however, the low $\mu_{\mathrm{FE}}$ and small driving current of approximately $10 \mu \mathrm{A}$ fail to meet the requirements for application in AMOLED displays.

To improve device performance, we adopt doped metal-oxide semiconductor of IGZO:Ti to form bilayer IGZO/IGZO:Ti channel structure. The Ti atom with higher bond enthalpy of Ti-O $(672 \mathrm{~kJ} / \mathrm{mole})$ [18] is preferred to getter oxygen atom in gate stack. The proposed oxygen gettering scheme to enhance device mobility is described as follows. The top IGZO layer incorporates $\mathrm{Ti}$ atoms that easily form strong bonds with oxygen. This

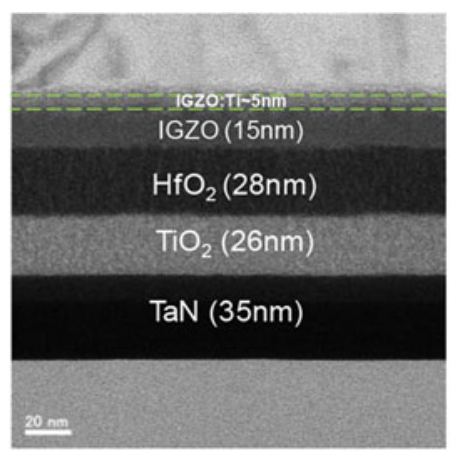

(a)

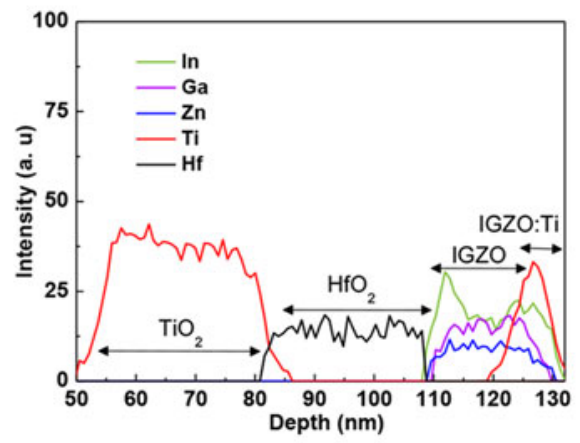

(b)

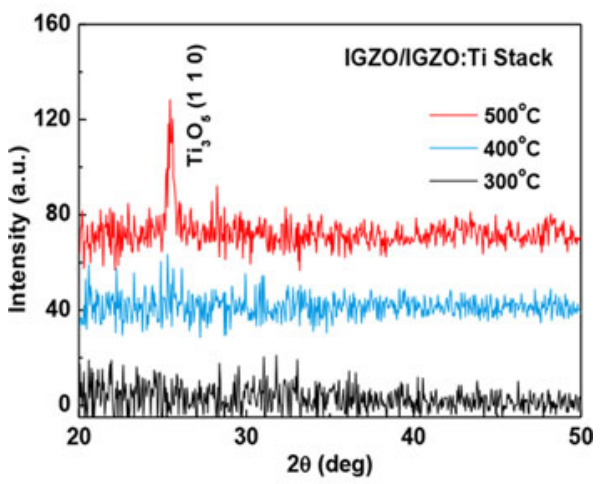

(c)

Fig. 3. (a) Cross-sectional view of TEM images and (b) EDX profile for IGZO/IGZO:Ti TFT. (c) XRD of bilayer IGZO/IGZO:Ti film with different annealed temperature of 300,400 , and $500{ }^{\circ} \mathrm{C}$.

driving force would result in the occurrence of oxygen gettering effect at high temperature, and thereby create more oxygen vacancies and electrons in active IGZO channel. The TEM photograph and EDX analysis of bilayer IGZO/IGZO:Ti TFT are shown in Fig. 3(a) and (b). From the analysis results, a small amount of Ti diffusion between the boundary of IGZO:Ti and bottom IGZO channel occurs after $300{ }^{\circ} \mathrm{C}$ annealing, but the 5-nm-thick IGZO:Ti layer and 15-nm-thick IGZO channel still can be defined by TEM image and EDX profile. Also, the $300{ }^{\circ} \mathrm{C}$ post annealing would not damage bottom gate stacks because the smooth interface between gate dielectric and IGZO/IGZO:Ti is clearly observed.

Fig. 3(c) shows XRD spectra of the IGZO/IGZO:Ti channel layers with different annealed temperatures. The XRD results 


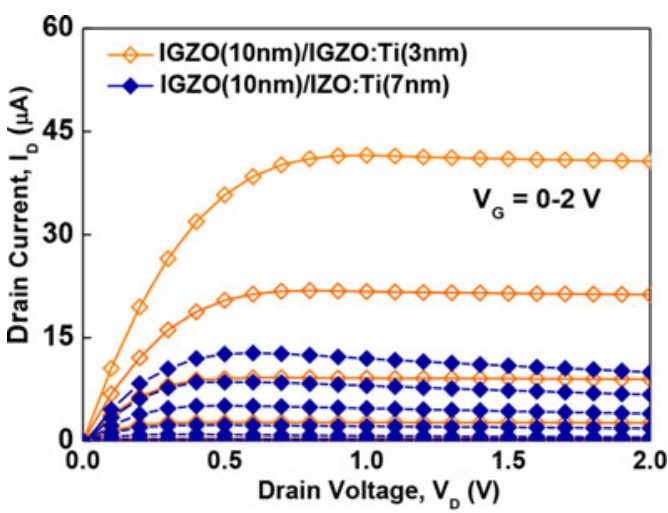

(a)

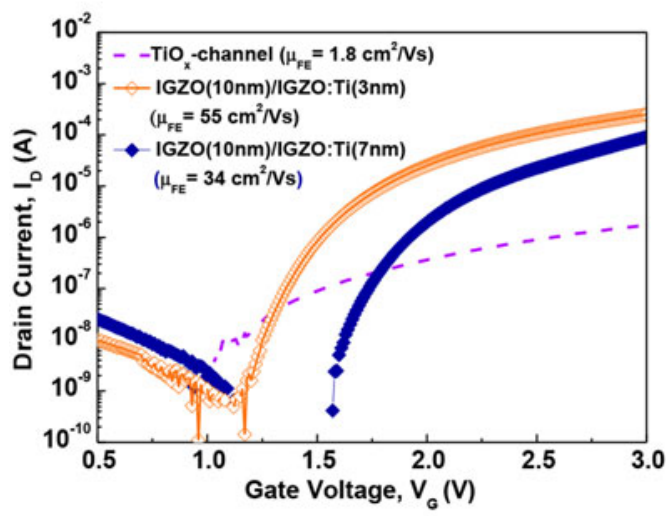

(b)

Fig. 4. (a) $I_{d}-V_{d}$ and (b) $I_{d}-V_{g}$ characteristics of bilayer IGZO/IGZO:Ti TFT devices with 10-nm top IGZO and different IGZO:Ti thicknesses.

reveal that an amorphous phase can be maintained at a process temperature of $400{ }^{\circ} \mathrm{C}$, but show a crystallized phase of $\mathrm{Ti}_{3} \mathrm{O}_{5}$ (110) at $500{ }^{\circ} \mathrm{C}$. The interface reaction and crystallized phase at high temperature would result in poor interface quality and influence off-state current and gate swing, which are not permitted for low-power TFT device. In the following section, we shall investigate the performance of metal-oxide TFT through the thickness change of IGZO:Ti and oxygen gettering to modify channel carriers concentration.

Besides, the n-type $\mathrm{TiO}_{x}$ has the advantages of being low cost and eco-friendly, which makes it potential candidate for semiconducting channels or gate dielectrics in next-generation displays. Thus, we also fabricated $\mathrm{TiO}_{x}$-channel TFT for performance comparison in this study. The $I_{d}-V_{d}$ and $I_{d}-V_{g}$ characteristics of single-layer $\mathrm{TiO}_{\mathrm{x}}$-channel TFT and bilayer IGZO(10 nm)/IGZO:Ti TFTs are shown in Fig. 4(a) and (b), respectively. Although the n-type $\mathrm{TiO}_{\mathrm{x}}$ material can be used as semiconducting channel, the on-current of $\sim 1 \mu \mathrm{A}$ is still small, which cannot meet the basic requirement of large current OLED. Compared with single-layer $\mathrm{TiO}_{x}$-channel TFTs, the bilayer IGZO/IGZO:Ti TFT with 10-nm-thick IGZO and 3-nm-thick IGZO:Ti exhibited a small $V_{T}$ of $1.5 \mathrm{~V}$ and a small $S S$ of $78 \mathrm{mV} /$ decade. Also, the large driving current of $250 \mu \mathrm{A}$ is $250 \times$ larger than that of $\mathrm{TiO}_{\mathrm{x}}$-channel TFTs. The extracted $\mu_{\mathrm{FE}}$ of $55 \mathrm{~cm}^{2} / \mathrm{Vs}$ is much higher than $1.8 \mathrm{~cm}^{2} / \mathrm{Vs}$ of $\mathrm{TiO}_{\mathrm{x}}$-channel

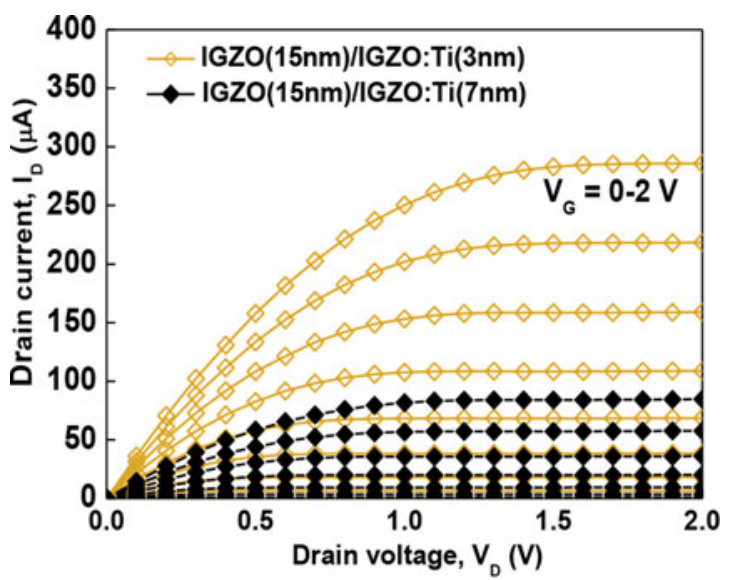

(a)

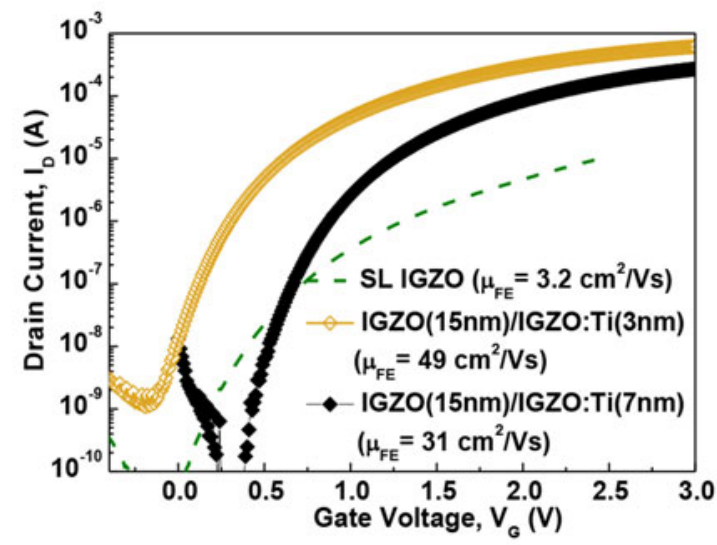

(b)

Fig. 5. (a) $I_{d}-V_{d}$ and (b) $I_{d}-V_{g}$ characteristics of bilayer IGZO/IGZO:Ti TFT devices with 15-nm top IGZO and different IGZO:Ti thicknesses.

TFTs. With the increase of IGZO:Ti thickness up to $7 \mathrm{~nm}$, the $S S$ ( $85 \mathrm{mV} / \mathrm{dec})$ is slightly degraded due to the increased thickness to dilute electric field across channel. The extracted high mobility of $34 \mathrm{~cm}^{2} / \mathrm{Vs}$ is also much better than single-layer IGZO TFT, indicating that the mobility enhancement is dependence of thickness modulation of IGZO:Ti layer. Therefore, we find that the absorbed oxygen capability of top IGZO:Ti dominates device mobility, but has less influence on $S S$ and $V_{T}$ under an appropriate thickness modulation.

To understand the relation of carrier mobility and bottom IGZO layer, we increase the thickness of bottom IGZO from $10 \mathrm{~nm}$ to $15 \mathrm{~nm}$. The $I_{d}-V_{d}$ and $I_{d}-V_{g}$ characteristics of bilayer IGZO(15 nm)/IGZO:Ti TFTs are shown in Fig. 5(a) and (b), respectively. Compared with conventional IGZO TFTs, the bilayer TFT incorporating a 3-nm-thick IGZO:Ti semiconducting layer exhibits a small $V_{T}$ of $0.36 \mathrm{~V}$ and a large driving current of $620 \mu \mathrm{A}$, which is $60 \times$ larger than that of single-layer IGZO TFT. The extracted $\mu_{\mathrm{FE}}$ of $49 \mathrm{~cm}^{2} / \mathrm{Vs}$ is also comparable to $\operatorname{IGZO}(10 \mathrm{~nm}) / \mathrm{IGZO}: \mathrm{Ti}$ TFT, but the $S S(121 \mathrm{mV} / \mathrm{dec})$ is apparently larger than that using thin 10-nm IGZO. Additionally, it is worth to note that the off-state current becomes larger and $V_{T}$ becomes smaller for the thicker bottom IGZO (15 nm) 


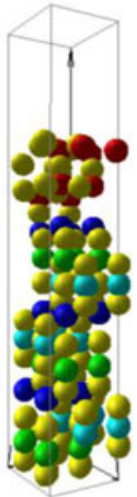

(a)

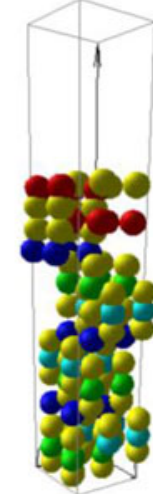

(b)
Fig. 6. Atomic structures of (a) $\mathrm{IGZO} / \mathrm{TiO}_{2}-\mathrm{TiO}_{2}$ and (b) $\mathrm{IGZO} / \mathrm{TiO}_{2}-\mathrm{TiO}$ with oxygen vacancy.

samples, indicating the increase of carrier density and degradation of gate control capability.

Fig. 5(b) shows the bilayer IGZO(15nm)/IGZO:Ti TFT using a thicker 7-nm-thick IGZO:Ti. The device performanceexhibits a low OFF current of $170 \mathrm{pA}$, large ON/OFF ratio of $3.9 \times 10^{6}$, and small $S S$ of $85 \mathrm{mV} /$ decade. Apparently, the ON/OFF ratio and OFF current are further improved by increasing the IGZO:Ti thickness from 3 to $7 \mathrm{~nm}$. The small gate swing was also supported by a low drive voltage $(<3 \mathrm{~V})$. Although the extracted $\mu_{\mathrm{FE}}$ of $31 \mathrm{~cm}^{2} / \mathrm{Vs}$ is smaller than the $49 \mathrm{~cm}^{2} / \mathrm{Vs}$ of 3-nm-thick IGZO:Ti sample, the $S S$ is much improved. It will be seen from this that the tradeoff between enhancing driving current and minimizing $S S$ is unavoidable because a thick IGZO:Ti layer may dilute the electric field across the bottom IGZO channel and result in low current driving capability. Thus, the IGZO:Ti capping layer not only acts as mobility booster, but also serves as a current modulator that improves gate swing and off-state current.

To further investigate oxygen gettering mechanism, we analyze electronic structure and transport mechanism in this bilayer amorphous oxide semiconductor. To analyze the thermodynamic stability between IGZO and $\mathrm{TiO}_{2}$, the formation energy was calculated by relaxing the $2 \times 2 \times 1$ supercell with constrained $\left(\mathrm{InGaZnO}_{4}\right)_{3}$. The unit cell was defined to contain three $\mathrm{In}$, three $\mathrm{Ga}$, three $\mathrm{Zn}$, and twelve $\mathrm{O}$ atoms. The interfaces were modeled with a superlattice, including one interface and a $17 \AA$ vacuum layer separating the periodic images along the $c$-axis. Subsequently, the first-principle calculations were carried out using the density-functional theory with the total energy plane-wave basis code, VASP [19], [20]. The exchange correlation energy was approximated with the generalized gradient approximation. The pseudopotential was based on the projected augmented wave method. Fig. 6(a) and (b) shows the relaxed atomic structures of In-terminated (In-ter) $\mathrm{IGZO} / \mathrm{TiO}_{2}-\mathrm{TiO}_{2}$ and $\mathrm{IGZO} / \mathrm{TiO}_{2}-\mathrm{TiO}$ with a missing oxygen (oxygen-vacancy structure), respectively.

The projected density of state (DOS) for each atom, In, Ga, $\mathrm{Zn}$, Ti, and $\mathrm{O}$, is shown in Figs. 7 and 8. The projected DOS

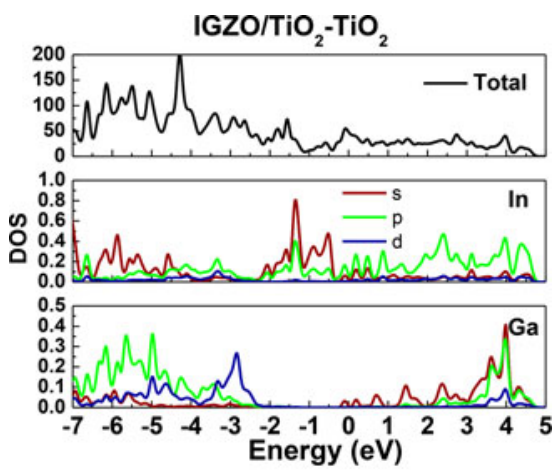

(a)

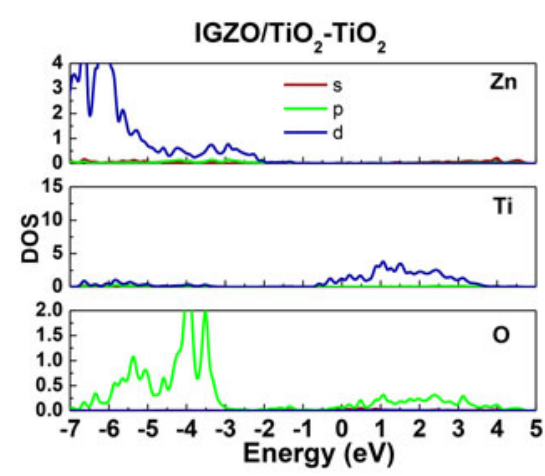

(b)

Fig. 7. Density of states of (a) total, In, Ga, (b) $\mathrm{Zn}$, Ti, and $\mathrm{O}$ atoms for $\mathrm{IGZO} / \mathrm{TiO}_{2}-\mathrm{TiO}_{2}$ film structure.

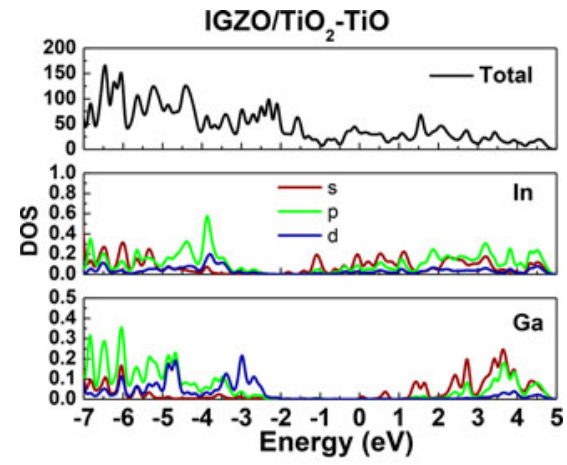

(a)

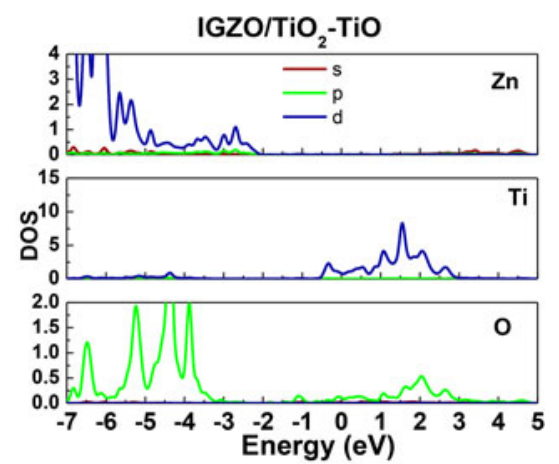

(b)

Fig. 8. Density of states of (a) total, In, Ga, (b) $\mathrm{Zn}, \mathrm{Ti}$ and $\mathrm{O}$ atoms for $\mathrm{IGZO} / \mathrm{TiO}_{2}-\mathrm{TiO}$ film structure with oxygen vacancy. 


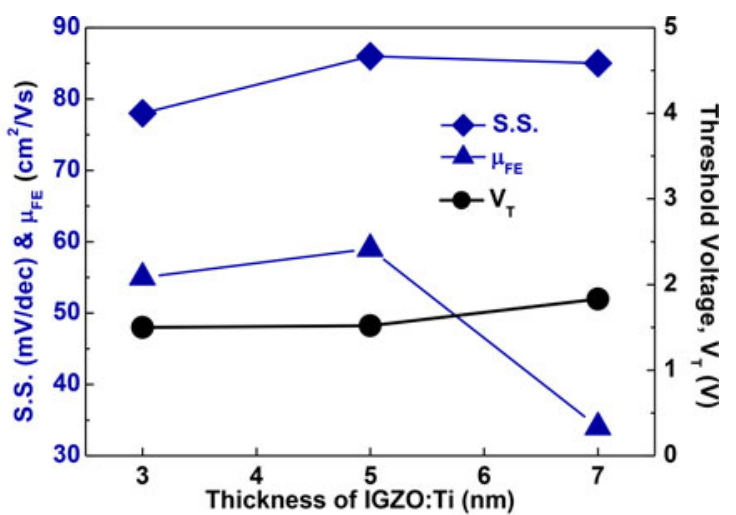

(a)

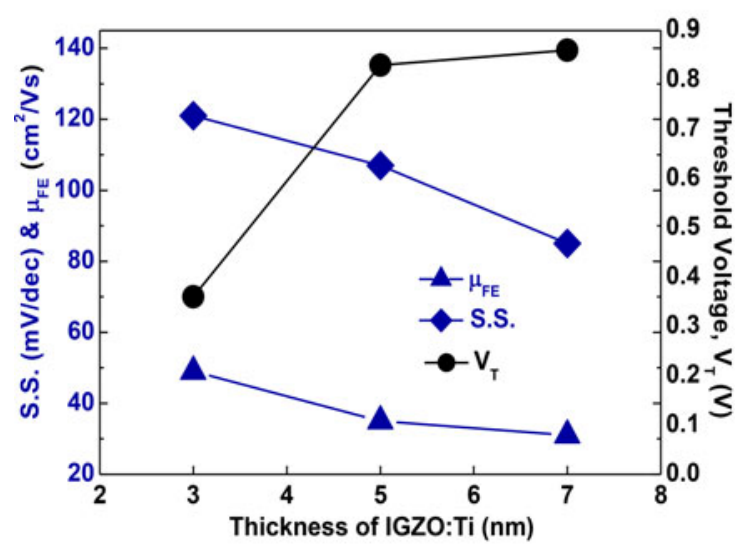

(b)

Fig. 9. $\mu_{\mathrm{FE}}, S S$, and $V_{T}$ as a function of IGZO:Ti thickness for (a) IGZO(10 nm)/IGZO:Ti and (b) IGZO(15 nm)/IGZO:Ti TFT devices.

illustrates that the valence band consists mainly of O-2p orbital characters, but the lowest conduction band mostly shows Ti-4d orbital characters. In $\mathrm{IGZO} / \mathrm{TiO}_{2}-\mathrm{TiO}$ system with oxygen vacancy, the conduction bands show stronger band dispersion in Ti-4d orbital characters. The localized electronic states near conduction band edge are also observed, which is associated with the defect vacancies. Besides, the total energy of $-5.85 \mathrm{eV} /$ atom for the $\mathrm{IGZO} / \mathrm{TiO}_{2}-\mathrm{TiO}$ system appeared to be lower than that of $-5.93 \mathrm{eV} /$ atom for the $\mathrm{IGZO} / \mathrm{TiO}_{2}-\mathrm{TiO}_{2}$ interface, indicating low thermodynamic stability in $\mathrm{IGZO} \mathrm{TiO}_{2}-\mathrm{TiO}$ system. Therefore, the charged $\mathrm{Ti}$ atom can serve as an oxygen getter that induces oxygen vacancies and increases carrier concentration in IGZO at high temperature.

Fig. 9 displays the summarized $I_{d}-V_{g}$ characteristics as a function of the IGZO:Ti capping layer thickness. Compared with metal-oxide TFTs that incorporate single-layer channels of $\mathrm{TiO}_{2}$ and IGZO, the dual-channel TFT that optimizes the thickness of the 5-nm-thick IGZO:Ti capping layer attains the best performance such as the lowest $S S$ ( $85 \mathrm{mV} /$ decade $)$ and the highest $\mu_{\mathrm{FE}}\left(49 \mathrm{~cm}^{2} / \mathrm{Vs}\right)$ at a low drive voltage $(<3 \mathrm{~V})$. However, with increasing IGZO:Ti thickness, a tradeoff on gate swing and mobility comes out. This is because an excessive IGZO:Ti thickness affects electron accumulation under an overdrive voltage, which results in low carrier concentration and small driving current. Such improvement primarily results from the IGZO:Ti capping layer, which exerts an oxygen gettering effect, simultaneously enhancing device mobility and improving gate swing.

\section{CONCLUSION}

A high-mobility IGZO TFT that operated at a low drive voltage $(<3 \mathrm{~V})$ was demonstrated in this study. The proposed bilayer IGZO/IGZO:Ti device demonstrated the lowest OFF current of $170 \mathrm{pA}$, the smallest $S S$ of $85 \mathrm{mV} /$ decade, and the highest $\mu_{\mathrm{FE}}$ of $49 \mathrm{~cm}^{2} / \mathrm{Vs}$; these favorable results were obtained by modulating the IGZO:Ti thickness. These bilayer TFTs using oxygen gettering scheme have potential applications in high-resolution displays.

\section{REFERENCES}

[1] R. Fardel, M. Nagel, F. Nuesch, T. Lippert, and A. Wokaun, "Fabrication of organic light-emitting diode pixels by laser-assisted forward transfer," Appl. Phys. Lett., vol. 91, p. 061103, Aug. 2007.

[2] C. D. Muller, A. Falcou, N. Reckefuss, M. Rojahn, V. Wiederhirn, P. Ruati, H. Frohne, O. Nuyken, H. Becker, and K. Meerholz, "Multicolour organic light-emitting displays by solution processing," Nature, vol. 421, no. 6925, pp. 829-833, Feb. 2003.

[3] S. Ju, J. Li, J. Liu, P. C. Chen, Y. G. Ha, F. Ishikawa, H. Chang, C. Zhou, A. Facchetti, D. B. Janes, and T. J. Marks, "Transparent active matrix organic light-emitting diode displays driven by nanowire transistor circuitry," Nano Lett., vol. 8, no. 4, pp. 997-1004, Dec. 2007.

[4] C. D. Dimitrakopoulos, S. Purushothaman, J. Kymissis, A. Callegari, and J. M. Shaw, "Low-voltage organic transistors on plastic comprising high-dielectric constant gate insulators," Science, vol. 283, no. 5403, pp. 822-824, Feb. 1999.

[5] P. Barquinha, A. M. Vila, G. Goncalves, L. Pereira, R. Martins, J. R. Morante, and E. Fortunato, "Gallium-indium-zinc-oxide-based thinfilm transistors: Influence of the source/drain material," IEEE Trans. Electron Devices, vol. 55, no. 4, pp. 954-960, Apr. 2008.

[6] K. Nomura, H. Ohta, K. Ueda, T. Kamiya, M. Hirano, and H. Hosono, "Thin-film transistor fabricated in single-crystalline transparent oxide semiconductor,' Science, vol. 300, pp. 1269-1272, May 2003.

[7] A. Suresh, P. Wellenius, and J. F. Muth, "High performance transparent thin film transistors based on indium gallium zinc oxide as the channel material," in Proc. IEDM Tech. Dig., Dec. 2007, pp. 587-590.

[8] M. J. Yu, Y. H. Yeh, C. C. Cheng, C. Y. Lin, G. T. Ho, B. C. M. Lai, C. M. Leu, T. H. Hou, and Y. J. Chan, "Amorphous InGaZnO thin-film transistors compatible with roll-to-roll fabrication at room temperature," IEEE Electron Device Lett., vol. 33, no. 1, pp. 47-49, Jan. 2012.

[9] C. H. Cheng, F. S. Yeh, and A. Chin, "Low-power high-performance nonvolatile memory on a flexible substrate with excellent endurance," $A d v$. Mater., vol. 23, no. 7, pp. 902-905, Feb. 2011.

[10] H. H. Hsu, C. Y. Chang, and C. H. Cheng, "A flexible IGZO thinfilm transistor with stacked $\mathrm{TiO}_{2}$-based dielectrics fabricated at room temperature," IEEE Electron Device Lett., vol. 34, no. 6, pp. 768-770, Jun. 2013.

[11] K. Nomura, H. Ohta, K. Ueda, T. Kamiya, M. Hirano, and H. Hosono, "Thin-film transistor fabricated in single-crystalline transparent oxide semiconductor," Science, vol. 300, no. 5623, pp. 1269-1272, May 2003.

[12] W. Lim, S. Kim, Y. Wang, J. Lee, D. Norton, S. Pearton, F. Ren, and I. Kravchenko, "Stable room temperature deposited amorphous InGaZnO4 thin film transistors," J. Vac. Sci. Technol. B, vol. 26, no. 3, pp. 959-962, May 2008.

[13] H. Cho, E. A. Douglas, B. P. Gila, V. Craciun, E. S. Lambers, F. Ren, and S. J. Pearton, "Band offsets in $\mathrm{HfO}_{2} / \mathrm{InGaZnO}_{4}$ heterojunctions," Appl. Phys. Lett., vol. 100, pp. 012105-1-012105-3, Jan. 2012.

[14] C. H. Cheng, S. H. Lin, K. Y. Jhou, W. J. Chen, C. P. Chou, F. S. Yeh, J. Hu, M. Huang, T. Arikado, S. P. McAlister, and A. Chin, "High density and low leakage current in $\mathrm{TiO}_{2}$ capacitors processed at $300{ }^{\circ} \mathrm{C}$," IEEE Electron Device Lett., vol. 29, no. 8, pp. 845-847, Aug. 2008.

[15] H. H. Hsu, C. Y. Chang, and C. H. Cheng, "Room-temperature flexible thin film transistor with high mobility," Current Appl. Phys., vol. 13, pp. 1459-1462, 2013. 
[16] H. H. Hsu, C. Y. Chang, C. H. Cheng, S. H. Yu, C. Y. Su, and C. Y $\mathrm{Su}$, "Fully room-temperature IGZO thin film transistors adopting stacked gate dielectrics on flexible polycarbonate substrate," Solid-State Electron., vol. 89, pp. 194-197, 2013.

[17] H. H. Hsu, C. Y. Chang, and C. H. Cheng, "Flexible IGZO TFT stacked $\mathrm{Y}_{2} \mathrm{O}_{3} / \mathrm{TiO}_{2} / \mathrm{Y}_{2} \mathrm{O}_{3}$ gate dielectrics grown at room temperature," Physica Status Solidi, vol. 7, pp. 285-288, 2013.

[18] C. H. Cheng, K. I. Chou, and A. Chin, "Gate-first $n$-MOSFET with a Sub-0.6-nm EOT gate stack,” Microelectron. Eng., vol. 109, pp. 35-38, Sep. 2013.
[19] G. Kresse and D. Joubert, "From ultrasoft pseudopotentials to the projector augmented-wave method," Phys. Rev. B, vol. 59, no. 3, pp. 1758-1775, Jan. 1999.

[20] G. Kresse and J. Furthmuller, "Efficiency of ab-initio total energy calculations for metals and semiconductors using a plane-wave basis set," Comput. Mater. Sci., vol. 6, no. 1, pp. 15-50, Mar. 1996.

Authors' photographs and biographies not available at the time of publication. 